\title{
ASPECTOS BIOLÓGICOS DE Thyrinteina arnobia (LEP.: GEOMETRIDAE) PROVENIENTES DE LAGARTAS CRIADAS EM FOLHAS DE Eucalyptus cloeziana OU DE Psidium guajava SOB CONDIÇÕES DE CAMPO ${ }^{1}$
}

\author{
Anderson Mathias Holtz² José Cola Zanuncio $^{3}$, Hamilton Gomes de Oliveira ${ }^{2}$, Angelo Pallini ${ }^{4}$, Jeanne Scardini \\ Marinho $^{5}$, Claudinei Lima Oliveira ${ }^{6}$ Tobias Baruc Moreira Pinon ${ }^{6}$
}

\begin{abstract}
RESUMO - Os plantios de Eucalyptus no Brasil podem sofrer danos por espécies nativas de insetos de diversas ordens, como Orthoptera, Coleoptera e Lepidoptera. Esses insetos podem alimentar-se tanto de mirtáceas brasileiras como goiabeira, gabirobeira, jabuticabeira, entre outras, como de espécies do gênero Eucalyptus. Entre os desfolhadores, destaca-se Thyrinteina arnobia (Stoll) (Lepidoptera: Geometridae) como o mais daninho dessa ordem para a eucaliptocultura brasileira. Este trabalho teve por objetivo avaliar aspectos biológicos de adultos de T. arnobia provenientes de lagartas alimentadas com plantas de eucalipto e, ou, goiabeira. Adultos deste inseto criados em folhas de eucalipto e, ou, de goiabeira apresentaram diferenças significativas para a maioria dos aspectos biológicos avaliados, exceto para a duração dos períodos de pré-oviposição, de oviposição e razão sexual. Assim, insetos herbívoros que vivem em hospedeiros filogeneticamente próximos ao eucalipto são capazes de causar danos consideráveis em reflorestamentos com espécies desse grupo, o que provavelmente ocorre pelo fato de elas estarem ainda em processo de adaptação a essa praga que atacaria o eucalipto, por estar fugindo da pressão exercida por barreiras físicas e químicas existentes nas mirtáceas nativas brasileiras.
\end{abstract}

Palavras-chave: Biologia, Thyrinteina arnobia, Eucalyptus cloeziana e Psidium guajava.

\section{BIOLOGICAL ASPECTS OF Thyrinteina arnobia (LEP.: GEOMETRIDAE) ADULTS ORIGINATED FROM CATERPILLARS REARED ON LEAVES OF Eucalyptus cloeziana OR Psidium guajava UNDER FIELD CONDITIONS}

\begin{abstract}
Eucalyptus plantations in Brazil may be damaged by native insects of many orders including Orthoptera, Coleoptera and Lepidoptera. These insects feed on Brazilian tree species of the family Myrtaceae to which the genus Eucalyptus belongs. The Lepidoptera Thyrinteina arnobia Stoll (Lepidoptera: Geometridae) is the most harmful defoliator of Eucalyptus in Brazil. The objective of this work was to evaluate biological aspects of T. arnobia adults originated from caterpillars fed on guava or eucalyptus plants. Adults of T. arnobia originated from caterpillars reared with eucalyptus or guava leaves presented significant differences for most biological aspects evaluated, except for duration of preoviposition and oviposition periods and sex ratio. This shows that species feeding on host plants related to eucalyptus can cause considerable damage in reforestation with species of this genus. T. arnobia insects are still undergoing an imposed adaptation process to eucalyptus plants and likely feed on these plants to escape from pressure by physical and chemical barriers of Brazilian native Myrtaceae.
\end{abstract}

Key words: Biology, Thyrinteina arnobia, Eucalyptus cloeziana, Psidium guajava

1 Recebido para publicação em 12.8.2002.

Aceito para publicação em 9.9.2003.

2 Eng.-Agrônomo, Estudante de Doutorado, Bolsista da CAPES e CNPq, respectivamente - Departamento de Entomologia da Universidade Federal de Viçosa - UFV, 36570-000 Viçosa-MG, <aholtz@insecta.ufv.br>, <hamilton@insecta.ufv.br>; ${ }^{3} \mathrm{Ph} . D$. ., Prof. Titular e Bolsista do CNPq, Eng. Florestal, Departamento de Biologia Animal - DBA-UFV, <zanuncio@ufv.br>; ${ }^{4}$ Ph.D., Prof. Adjunto, Entomologia - DBA-UFV, <pallini@ufv.br>; ${ }^{5}$ Estudante de Agronomia, Bolsista de Iniciação Científica do CNPq, <jeanne@insecta.ufv.br>; ${ }^{6}$ Estudante de Eng. Florestal, Bolsista de Iniciação Científica do CNPq, <claudinei@insecta.ufv.br> 


\section{INTRODUÇÃO}

As Myrtaceae nativas do Brasil (goiabeira, gabirobeira etc.) abrigam uma entomofauna indígena que sobrevive nesses hospedeiros (Anjos et al., 1987). O incremento da eucaliptocultura, como cultura exótica no território nacional, tem propiciado um número crescente de espécies indígenas de lepidópteros, provenientes de mirtáceas nativas que passaram a alimentar-se de plantas de eucalipto (Santos et al., 2000), como Eupseudosoma aberrans e Eupseudosoma involuta (Arctiidae), Automeris spp., Eacles imperialis e Hylesia spp. (Saturniidae), Sabulodes caberata, Thyrinteina arnobia e Oxydia vesulia (Geometridae) (Anjos et al., 1987). Espécies de insetos, de hospedeiros filogeneticamente próximos ao eucalipto, são capazes de causar danos consideráveis em reflorestamentos por estarem em processo de adaptação ao Eucalyptus spp. (Santos et al., 1989; Zanuncio et al., 1990).

O gênero Eucalyptus, com mais de 600 espécies e variedades, foi introduzido no Brasil em 1824 e passou a ser plantado com fins comerciais em 1904 (Zanuncio et al., 1991). Porém, somente a partir da década de 70, devido à expansão da indústria siderúrgica, que utiliza carvão vegetal, e da indústria de papel e celulose, ocorreu a implantação de grandes maciços florestais no Brasil (Santos et al., 2000). Assim, além de exótica, a eucaliptocultura é recente no País e insetos herbívoros como T. arnobia, que antes se alimentavam de mirtáceas nativas como a goiabeira, passaram a atacar o eucalipto, causando sérios prejuízos a esta essência florestal (Anjos et al., 1987). A importância desse gênero de herbívoro pode ser mostrada pelo fato de $T$. arnobia ser a lagarta desfolhadora de eucalipto mais estudada no Brasil e estar presente na maioria das áreas cultivadas com esta cultura. Além desta, outras espécies como Thyrinteina leucoceraea (Lepidoptera: Geometridae) também são importantes para a eucaliptocultura (Zanuncio et al., 1997).

O objetivo desta pesquisa foi estudar aspectos biológicos de adultos de T. arnobia provenientes de lagartas criadas com folhas de eucalipto (Eucalyptus cloeziana F. Muell.) ou de goiabeira (Psidium guajava L.), em condições de campo.

\section{MATERIAL E MÉTODOS}

\subsection{Instalações, Criação e Manutenção de T. arnobia}

Adultos provenientes de coleta, em campo, em plantas de eucalipto foram utilizados para criação e

R. Árvore, Viçosa-MG, v.27, n.6, p.897-901, 2003 manutenção de T. arnobia. Os adultos foram levados para o Laboratório de Entomologia Florestal da Universidade Federal de Viçosa (UFV) e mantidos em sala climatizada com temperatura de $25 \pm 2{ }^{\circ} \mathrm{C}$, fotofase de 12 horas e umidade relativa de $60 \pm 10 \%$. Em seguida, foram separados em casais e acondicionados em potes plásticos (500 ml), com tampa plástica com um furo no centro, o qual foi vedado com uma tela de malha fina, tipo organza. Dentro desses potes foram colocadas tiras de papel presas à tampa, para que nelas fossem efetuadas as posturas.

Logo após a eclosão, as lagartas de T. arnobia foram colocadas em sacos de tecido tipo organza $(0,70 \mathrm{x}$ 0,40 m), envolvendo galhos de plantas de eucalipto e, ou, de goiabeira com folhas sem danos. Essas lagartas foram mantidas por quatro gerações, em cada espécie de planta (eucalipto e, ou, goiaba). Desta forma, foram obtidas duas populações de T. arnobia, conforme a dieta recebida.

Nos primeiros estádios, as lagartas de T. arnobia foram removidas para outros galhos (eucalipto e, ou, goiaba) com sacos de organza, quando as folhas começavam a secar. A troca de galhos foi mais freqüente a partir do quarto estádio, quando as lagartas alimentam-se mais vorazmente.

\subsection{Aspectos Biológicos de T. arnobia}

Aspectos da fase adulta de T. arnobia foram estudados com 20 casais para cada dieta (eucalipto e, ou, goiabeira), sendo cada repetição composta por um casal de T. arnobia acondicionado em pote plástico (conforme descrição anterior). Foram colocadas tiras de papel presas à tampa dos copos, para facilitar a oviposição. Foram observados os períodos de pré-oviposição e de oviposição, os números de ovos, de posturas, de lagartas e de óvulos retidos no abdômen por fêmea, além do período de incubação dos ovos e a longevidade dos adultos. Foram observados a duração do período de pré-pupa e o peso e a viabilidade da fase de pupa de 100 lagartas por hospedeiro.

Os dados obtidos foram analisados pelo teste $\mathrm{F}$ a $5 \%$ de significância.

\section{RESULTADOS E DISCUSSÃO}

Adultos de T. arnobia apresentaram diferenças significativas para a maioria dos parâmetros analisados, entre aqueles obtidos de lagartas criadas em folhas de 
eucalipto ou de goiabeira (Quadro 1) $(\mathrm{p}<0,05)$, exceto para a duração dos períodos de pré-oviposição, de oviposição e razão sexual, mostrando que a goiabeira não é um bom hospedeiro para o desenvolvimento populacional de T. arnobia, possivelmente pelos mecanismos de defesa deste hospedeiro (defesas químicas que agem diretamente sobre o herbívoro), que interferem negativamente no desempenho desse inseto.

Fêmeas de T. arnobia criadas em eucalipto apresentaram maior número de ovos e de lagartas por postura que aquelas criadas com folhas de goiabeira (Quadro 1) (Figura 1a e 1b). Zanuncio et al. (1990) comentam que essa e outras espécies de insetos encontram-se em processo de adaptação a plantas de eucalipto, ao fugirem da pressão exercida por barreiras físicas e químicas que, possivelmente, existem nas mirtáceas nativas brasileiras. As barreiras químicas nas plantas incluem a produção de toxinas que atuam principalmente na redução da digestibilidade pelo inseto, afetando, desta forma, o seu desempenho no hospedeiro e seu desenvolvimento na população atual, bem como nas suas próximas gerações (Price, 1997; Mauricio \& Rausher, 1997).

A qualidade nutricional e a digestibilidade das plantas são críticas para os herbívoros, devendo ser ressaltado que os compostos secundários presentes em muitas plantas reduzem a disponibilidade de proteínas aos herbívoros (Jãremo, 1999). Por exemplo, os taninos armazenados em vacúolos nas folhas de carvalho e de outras plantas combinam-se com as proteínas das folhas e enzimas digestivas nos intestinos dos herbívoros, o que dificulta a digestão das proteínas (Panizzi \& Parra, 1991). Deste modo, os taninos podem diminuir, consideravelmente, a absorção de nutrientes pelos herbívoros e reduzir o número de seus descendentes (Berennbaum \& Zangerl, 1996).

A porcentagem de eclosão de lagartas e o número de lagartas por postura de T. arnobia foram maiores para adultos oriundos de lagartas alimentadas com eucalipto (Figura 2a) do que para aquelas alimentadas com goiabeira (Quadro 1, Figura 1b). Santos et al. (2000) mostraram que T. arnobia se desenvolveu melhor em plantas de goiaba que naquelas de eucalipto. Esta diferença pode estar relacionada com o período de adaptação do inseto ao novo hospedeiro, pois os autores mediram as taxas de mortalidade utilizando diretamente posturas de T. arnobia coletadas em eucalipto no campo, sem manter os insetos provenientes dessas posturas, por algumas gerações, no novo hospedeiro (goiaba), como foi realizado no presente trabalho. Não ocorrendo o período de adaptação, os herbívoros podem ser eficientes em alguns aspectos biológicos sobre o novo hospedeiro, em uma primeira geração. Porém, a partir da segunda e das gerações subseqüentes, a população do herbívoro pode ser afetada pelos compostos químicos da planta (Berenbaum \& Zangerl, 1996).

Quadro 1 - Aspectos biológicos de Thyrinteina arnobia (Lepidoptera: Geometridae) provenientes de lagartas criadas com Eucalyptus cloeziana (eucalipto) ou Psidium guajava (goiabeira) em condições de campo. Viçosa, Minas Gerais

Table 1 - Biological aspects of Thyrinteina arnobia (Lepidoptera: Geometridae) originated from caterpillars reared on Eucalyptus cloeziana (eucalyptus) or Psidium guajava (guava) under field conditions. Viçosa, Minas Gerais, Brazil

\begin{tabular}{|c|c|c|c|c|c|c|}
\hline Parâmetro & \multicolumn{3}{|c|}{ Eucalipto } & \multicolumn{3}{|c|}{ Goiabeira } \\
\hline Pré-oviposição (dias) & 1,55 & \pm & $0,39 a$ & 1,25 & \pm & $0,44 \mathrm{a}$ \\
\hline Oviposição (dias) & 4,10 & \pm & $1,41 \mathrm{a}$ & 5,25 & \pm & $2,43 \mathrm{a}$ \\
\hline Ovos Totais/Fêmea & $1.059,20$ & \pm & $412,34 \mathrm{a}$ & 706,01 & \pm & $429,13 \mathrm{~b}$ \\
\hline Total de Lagartas/Fêmea & 834,85 & \pm & $322,28 \mathrm{a}$ & 477,72 & \pm & $293,16 \mathrm{~b}$ \\
\hline Incubação (dias) & 10,20 & \pm & $0,92 \mathrm{~b}$ & 12,28 & \pm & $0,60 \mathrm{a}$ \\
\hline Óvulos Retidos no Abdome & 22,20 & \pm & $41,71 \mathrm{~b}$ & 196,20 & \pm & $230,87 \mathrm{a}$ \\
\hline Longevidade de Adultos (dias) & 7,05 & \pm & $2,26 b$ & 9,30 & \pm & $2,11 \mathrm{a}$ \\
\hline Período de Pré-pupa & 2,28 & \pm & $0,45 \mathrm{~b}$ & 2,67 & \pm & $0,67 \mathrm{a}$ \\
\hline Peso da Pupa (mg) (?) & 294,60 & \pm & $0,02 \mathrm{a}$ & 224,60 & \pm & $0,05 \mathrm{~b}$ \\
\hline Peso da Pupa (mg) (?) & 776,90 & \pm & $0,13 \mathrm{a}$ & 635,50 & \pm & $0,18 \mathrm{~b}$ \\
\hline Viabilidade Pupal (\%) & 78,87 & \pm & $1,32 \mathrm{a}$ & 25,00 & \pm & $1,17 \mathrm{~b}$ \\
\hline Razão Sexual & 0,40 & \pm & $0,02 \mathrm{a}$ & 0,43 & \pm & $0,06 \mathrm{a}$ \\
\hline Ciclo Pupal (dias) & 9,48 & \pm & $1,08 \mathrm{~b}$ & 10,95 & \pm & $1,08 \mathrm{a}$ \\
\hline
\end{tabular}

Médias seguidas de mesma letra na linha não diferem entre si pelo teste $F(p>0,05)$. 

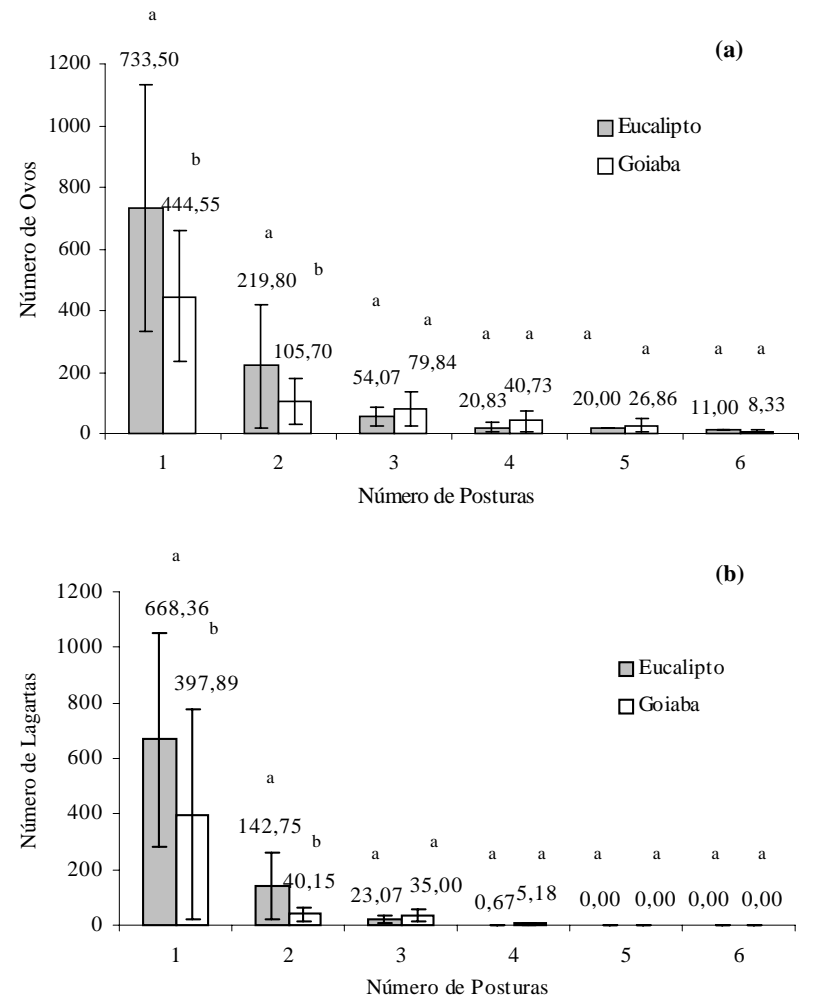

Figura 1 - Número de ovos (a) e de lagartas (b) por fêmea de Thyrinteina arnobia (Lepidoptera: Geometridae) provenientes de lagartas criadas com Eucalyptus cloeziana (eucalipto) ou Psidium guajava (goiabeira). Médias seguidas de pelo menos uma mesma letra nas barras em cada postura não diferem entre si pelo teste de $\mathrm{F}$, a $5 \%$ de significância.

Figure 1 - Number of eggs (a) and caterpillars (b) for each female of Thyrinteina arnobia (Lepidoptera: Geometridae) originated from caterpillars reared on Eucalyptus cloeziana (eucalyptus) or Psidium guajava (guava). Averages followed by, at least, one same letter in the bars per each egg mass, do not differ between each other by the F test at 5\% probability.

A menor aptidão de T. arnobia por plantas de goiaba pode ter ocorrido pela atuação de sistemas de defesa química dessas plantas. Maurício \& Rausher (1997) mostraram que barreiras químicas incluem a produção de toxinas que atuam, principalmente, na redução da digestibilidade de insetos e, portanto, afetam seu desempenho no hospedeiro e seu desenvolvimento na população atual e nas gerações futuras. A maioria das defesas químicas de plantas é constitutiva e evita ataques de herbívoros, mas algumas dessas espécies conseguem suplantar e consumir tais plantas, embora com prejuízo para seu desempenho e desenvolvimento (Karban \& Baldwin, 1997).

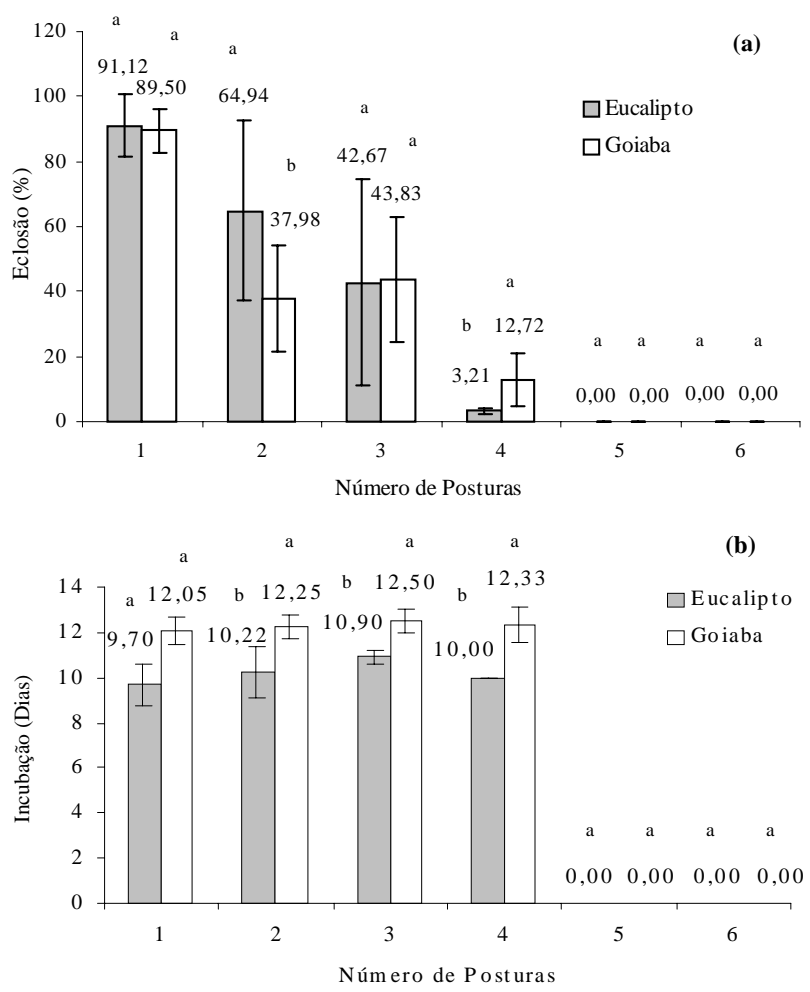

Figura 2 - Porcentagem de eclosão de lagartas (a) e período de incubação de ovos (b) de Thyrinteina arnobia (Lepidoptera: Geometridae) de lagartas criadas com Eucalyptus cloeziana (eucalipto) ou Psidium guajava (goiabeira). Médias seguidas de pelo menos uma mesma letra nas barras em cada postura não diferem entre si pelo teste de $\mathrm{F}$, a $5 \%$ de significância.

Figure 2-Percentage of eclosion of caterpillars (a) and period of egg incubation (b) of Thyrinteina arnobia (Lepidoptera: Geometridae) from caterpillars reared on Eucalyptus cloeziana (eucalyptus) or Psidium guajava (guava). Averages followed by, at least, one same letter in the bars in each egg mass, do not differ between each other, by the F test at $5 \%$ probability.

O período de incubação dos ovos de T. arnobia foi menor para adultos originados de lagartas alimentadas com eucalipto do que com goiabeira (Figura 2B), enquanto a longevidade de adultos foi maior para insetos provenientes de goiabeira (Quadro 1). Tal fato mostra que é importante comparar os parâmetros biológicos de insetos herbívoros e analisar a sua capacidade de mantêlos ou não, quando se desenvolvem sobre espécies diferentes (Meijden et al., 1988; Prins \& Verkaar, 1992). O fato de os parâmetros não serem mantidos sobre uma determinada espécie de planta, mesmo que os insetos tenham sido encontrados sobre ela, pode ser uma 
indicação de que estes insetos não estão adaptados a essa espécie de planta (Welter \& Steggal, 1993; Lehtilã \& Syrjnanen, 1995; Rosenthal \& Welter, 1995).

\section{CONCLUSÕES}

T. arnobia apresenta dificuldade em se desenvolver e estabelecer suas populações em plantas de goiabeira (mirtácea nativa), mas, por outro lado, esta espécie de herbívoro consegue desenvolver e manter suas populações em plantas de eucalipto (mirtácea exótica). Uma hipótese para essa dificuldade poderia ser a atuação de mecanismos de defesa (defesas químicas que interferem negativamente no desempenho do inseto, o que afeta a biologia e contribui para redução do vigor dos indivíduos da espécie estudada). Além disto, em plantas de eucalipto, as possíveis barreiras químicas presentes podem ainda ser pouco efetivas devido ao curto período de tempo de exposição a T. arnobia. Portanto, seria interessante a realização de pesquisas sobre os compostos químicos que podem estar atuando em eucalipto e em goiabeira.

\section{AGRADECIMENTO}

Ao Conselho Nacional de Desenvolvimento Científico e Tecnológico (CNPq), à Coordenação de Aperfeiçoamento de Pessoal de Nível Superior (CAPES) e à Fundação de Amparo à Pesquisa do Estado de Minas Gerais (FAPEMIG).

\section{REFERÊNCIAS BIBLIOGRÁFICAS}

ANJOS, N.; SANTOS, G. P.; ZANUNCIO, J. C. A lagartaparda, Thyrinteina arnobia Stoll, 1782 (Lepidoptera: Geometridae) desfolhadora de eucaliptos. Viçosa-MG: EPAMIG. 1987. 56 p. (Boletim Técnico, 25)

BERENNBAUM, M. R.; ZANGERL, A. R. Constraints on chemical coevolution: wild parsnips and the parsnip webworn. Evolution, v. 40, n. 3, p. 1215-1228, 1996.

JÃREMO, J. Plant adaptations to herbivory: mutualistic versus antagonistic coevolution. Oikos, v. 84, n. 1, p. 313320, 1999.

KARBAN, R.; BALDWIN, I. T. Induced responses to herbivory. Chicago, University of Chicago Press, 1997. $275 \mathrm{p}$.

LEHTILÃ, K. P.; SYRJANEN, K. Compensatory responses of two Melampyrum species after damage. Functional Ecology, v. 9, n. 1, p. 511-517, 1995.
MAURICIO, R.; RAUSHER, M. D. Variation in the defense strategies of plants: are resistance and tolerance mutually exclusive? Ecology, v. 78, n. 4, p. 1301-1311, 1997.

PANIZZI, A. R.; PARRA, J. R. P. Ecologia nutricional de insetos e suas aplicações no manejo de pragas. São Paulo, Manole, 1991. 391 p.

PRICE, P. W. Insect ecology. New York: John Wiley \& Sons, 1997. 874 p.

PRINS, A. H.; VERKAAR, H. J. Defoliation: of physiological and morphological responses lead to (over) compensation? In: AYRES, P. G. (Ed.) Pests and pathogens: plant responses to foliar attack. Oxford: Bios Scientific, 1997. p. 13-31.

ROSENTHAL, J. P.; WELTER, S. C. Tolerance to herbivory by a stem boring caterpillar in architecturally distinct maizes and wild relatives. Oecologia, v. 102, n. 11, p. 146-155, 1995.

SANTOS, G. P.; ANJOS, N.; ZANUNCIO, J. C. Biologia de Eustema sericea Schaus, 1922 (Lepidoptera: Notodontidae), desfolhadora de sobrasil, Colubrina rufa (Rhamnaceae). Anais da Sociedade Entomológica do Brasil, v. 18, n. 1, p. 247-256, 1989.

SANTOS, G. P.; ZANUNCIO, T. V.; ZANUNCIO, J. C. Desenvolvimento de Thyrinteina arnobia Stoll (Lepidoptera: Geometridae) em folhas de Eucalyptus urophylla e Psidium guajava. Anais da Sociedade Entomológica do Brasil, v. 29, n. 1, p. 13-22, 2000.

VAN DER MEIJDEN, E.; WIJN, M.; VERKAAR, H. J. Defense and regrowth, alternative plant strategies in the struggle against herbivores. Oikos, v. 51, n. 1, p. 355-363, 1988.

WELTER, S. C.; STEGGAL, J. W. Contrasting the tolerance of wild and domesticated tomatoes to herbivory: agroecological implications. Ecological Apple, v. 3, n. 1, p. 271-278, 1993.

ZANUNCIO, J. C. et al. Levantamento e flutuação populacional de lepidópteros associados à eucaliptocultura: V- Região de Belo Oriente, MG. Junho de 1986 a maio de 1987. Revista Árvore, v. 1, n. 14, p. 35-44, 1990.

ZANUNCIO, J. C. et al. Levantamento e flutuação populacional de lepidópteros associados à eucaliptocultura: VIII - Região de Belo Oriente, Minas Gerais, junho de 1989 a maio de 1990. Revista Árvore, v. 15, n. 1, p. 83-93, 1991.

ZANUNCIO, T. V. et al. Morfologia e bionomia de Thyrinteina leucoceraea Rindge (Lep., Geometridae) alimentadas com Eucalyptus urophylla. Revista Brasileira de Entomologia, v. 41, n. 1, p. 5-8, 1997.

R. Árvore, Viçosa-MG, v.27, n.6, p.897-901, 2003 